\title{
The Story of Riemann's Moduli Space
}

\section{by Lizhen $\mathrm{Ji}^{*}$}

\begin{abstract}
Compact Riemann surfaces and projective algebraic curves over $\mathbb{C}$ are two realizations of the same class of objects. Their moduli space was first introduced by Riemann and is one of the central objects of the contemporary mathematics and mathematical physics. In this note, we explain the birth and evolution of Riemann's moduli space by emphasizing several points which might not be so well-known to the general mathematicians and possibly even to some mathematicians who are interested in either complex analytic or algebraic geometric theories of moduli spaces. For example, who was the first to formulate the moduli problem precisely? What is the meaning of moduli spaces? Who was the first to give complex analytic and algebraic variety structures to Riemann's moduli space? Why did they study these problems? How was Riemann's moduli space first used? And why did Riemann choose and use the word moduli? Why did Teichmüller study Teichmüller space? What were Teichmüller's contributions to Riemann's moduli space? What were Teichmüller's contributions to algebraic geometry?
\end{abstract}

\section{Contents}

1 Introduction . . . . . . . . . . . . . . 46

2 Definitions . . . . . . . . . . . . . . 47

3 Riemann's Count of Moduli for Riemann Sur-

faces . . . . . . . . . . . . . . . . 49

* Department of Mathematics, University of Michigan, Ann Arbor, Mich., U.S.A.

E-mail: lji@umich.edu
4 Klein's Booklet on Riemann's Work and the Method of Continuity to Prove the Uniformization Theorem by Klein and Poincaré . 50

5 Teichmüller's Work on Moduli Space $\mathcal{M}_{g} \ldots 52$ 5.1 Teichmüller's Rigorous Formulation of and Results on Riemann's Moduli Space 52

5.2 Why Did Teichmüller Study Teichmüller Space and Riemann's Moduli Spaces? . . 57

6 Siegel Upper Half Space and Weil's Work on Weil-Petersson Metric . . . . . . . . . . . . 61

7 Grothendieck's Papers at Cartan Seminar . . . 62

8 Baily Compactification, Algebraic Structure on $\mathcal{M}_{g}$, and Torelli Morphism . . . . . . . . 64

9 Mumford's Work on $\mathcal{M}_{g}$ and Moduli Spaces . 67 10 Ahlfors and Bers' Works on Complex Analytic Geometry on Moduli Spaces . . . . . . . . 69 11 Meanings of Moduli in Moduli Spaces . . . . 70 Acknowledgments . . . . . . . . . . . . . 71 References . . . . . . . . . . . . . . . . . . . 71

\section{Introduction}

Mathematics is interesting and complicated. It also has interesting and complicated stories, which are often intriguing. Heroes can be mathematicians, or mathematical concepts and results. But interaction between them is probably more interesting.

In this article, we will tell the story of Riemann's moduli space. There are several reasons for choosing this topic. First, it had an honorable origin in the masterpiece "The theory of Abelian functions" by Riemann in 1857 [65, pp. 79-134]. Second, Riemann's moduli space of Riemann surfaces has been attracting more and more of people's attention, and research on it will probably continue for a long time 


\section{The Story of Riemann's Moduli Space}

\section{by Lizhen $\mathrm{Ji}^{*}$}

\begin{abstract}
Compact Riemann surfaces and projective algebraic curves over $\mathbb{C}$ are two realizations of the same class of objects. Their moduli space was first introduced by Riemann and is one of the central objects of the contemporary mathematics and mathematical physics. In this note, we explain the birth and evolution of Riemann's moduli space by emphasizing several points which might not be so well-known to the general mathematicians and possibly even to some mathematicians who are interested in either complex analytic or algebraic geometric theories of moduli spaces. For example, who was the first to formulate the moduli problem precisely? What is the meaning of moduli spaces? Who was the first to give complex analytic and algebraic variety structures to Riemann's moduli space? Why did they study these problems? How was Riemann's moduli space first used? And why did Riemann choose and use the word moduli? Why did Teichmüller study Teichmüller space? What were Teichmüller's contributions to Riemann's moduli space? What were Teichmüller's contributions to algebraic geometry?
\end{abstract}

\section{Contents}

1 Introduction . . . . . . . . . . . . . . 46

2 Definitions . . . . . . . . . . . . . . 47

3 Riemann's Count of Moduli for Riemann Sur-

faces . . . . . . . . . . . . . . . . 49

* Department of Mathematics, University of Michigan, Ann Arbor, Mich., U.S.A.

E-mail: lji@umich.edu
4 Klein's Booklet on Riemann's Work and the Method of Continuity to Prove the Uniformization Theorem by Klein and Poincaré . 50

5 Teichmüller's Work on Moduli Space $\mathcal{M}_{g} \ldots 52$ 5.1 Teichmüller's Rigorous Formulation of and Results on Riemann's Moduli Space 52

5.2 Why Did Teichmüller Study Teichmüller Space and Riemann's Moduli Spaces? . . 57

6 Siegel Upper Half Space and Weil's Work on Weil-Petersson Metric . . . . . . . . . . . . 61

7 Grothendieck's Papers at Cartan Seminar . . . 62

8 Baily Compactification, Algebraic Structure on $\mathcal{M}_{g}$, and Torelli Morphism . . . . . . . . 64

9 Mumford's Work on $\mathcal{M}_{g}$ and Moduli Spaces . 67 10 Ahlfors and Bers' Works on Complex Analytic Geometry on Moduli Spaces . . . . . . . . 69 11 Meanings of Moduli in Moduli Spaces . . . . 70 Acknowledgments . . . . . . . . . . . . . 71 References . . . . . . . . . . . . . . . . . . . 71

\section{Introduction}

Mathematics is interesting and complicated. It also has interesting and complicated stories, which are often intriguing. Heroes can be mathematicians, or mathematical concepts and results. But interaction between them is probably more interesting.

In this article, we will tell the story of Riemann's moduli space. There are several reasons for choosing this topic. First, it had an honorable origin in the masterpiece "The theory of Abelian functions" by Riemann in 1857 [65, pp. 79-134]. Second, Riemann's moduli space of Riemann surfaces has been attracting more and more of people's attention, and research on it will probably continue for a long time 


\section{The Story of Riemann's Moduli Space}

\section{by Lizhen $\mathrm{Ji}^{*}$}

\begin{abstract}
Compact Riemann surfaces and projective algebraic curves over $\mathbb{C}$ are two realizations of the same class of objects. Their moduli space was first introduced by Riemann and is one of the central objects of the contemporary mathematics and mathematical physics. In this note, we explain the birth and evolution of Riemann's moduli space by emphasizing several points which might not be so well-known to the general mathematicians and possibly even to some mathematicians who are interested in either complex analytic or algebraic geometric theories of moduli spaces. For example, who was the first to formulate the moduli problem precisely? What is the meaning of moduli spaces? Who was the first to give complex analytic and algebraic variety structures to Riemann's moduli space? Why did they study these problems? How was Riemann's moduli space first used? And why did Riemann choose and use the word moduli? Why did Teichmüller study Teichmüller space? What were Teichmüller's contributions to Riemann's moduli space? What were Teichmüller's contributions to algebraic geometry?
\end{abstract}

\section{Contents}

1 Introduction . . . . . . . . . . . . . . 46

2 Definitions . . . . . . . . . . . . . . 47

3 Riemann's Count of Moduli for Riemann Sur-

faces . . . . . . . . . . . . . . . . 49

* Department of Mathematics, University of Michigan, Ann Arbor, Mich., U.S.A.

E-mail: lji@umich.edu
4 Klein's Booklet on Riemann's Work and the Method of Continuity to Prove the Uniformization Theorem by Klein and Poincaré . 50

5 Teichmüller's Work on Moduli Space $\mathcal{M}_{g} \ldots 52$ 5.1 Teichmüller's Rigorous Formulation of and Results on Riemann's Moduli Space 52

5.2 Why Did Teichmüller Study Teichmüller Space and Riemann's Moduli Spaces? . . 57

6 Siegel Upper Half Space and Weil's Work on Weil-Petersson Metric . . . . . . . . . . . . 61

7 Grothendieck's Papers at Cartan Seminar . . . 62

8 Baily Compactification, Algebraic Structure on $\mathcal{M}_{g}$, and Torelli Morphism . . . . . . . . 64

9 Mumford's Work on $\mathcal{M}_{g}$ and Moduli Spaces . 67 10 Ahlfors and Bers' Works on Complex Analytic Geometry on Moduli Spaces . . . . . . . . 69 11 Meanings of Moduli in Moduli Spaces . . . . 70 Acknowledgments . . . . . . . . . . . . . 71 References . . . . . . . . . . . . . . . . . . . 71

\section{Introduction}

Mathematics is interesting and complicated. It also has interesting and complicated stories, which are often intriguing. Heroes can be mathematicians, or mathematical concepts and results. But interaction between them is probably more interesting.

In this article, we will tell the story of Riemann's moduli space. There are several reasons for choosing this topic. First, it had an honorable origin in the masterpiece "The theory of Abelian functions" by Riemann in 1857 [65, pp. 79-134]. Second, Riemann's moduli space of Riemann surfaces has been attracting more and more of people's attention, and research on it will probably continue for a long time 


\section{The Story of Riemann's Moduli Space}

\section{by Lizhen $\mathrm{Ji}^{*}$}

\begin{abstract}
Compact Riemann surfaces and projective algebraic curves over $\mathbb{C}$ are two realizations of the same class of objects. Their moduli space was first introduced by Riemann and is one of the central objects of the contemporary mathematics and mathematical physics. In this note, we explain the birth and evolution of Riemann's moduli space by emphasizing several points which might not be so well-known to the general mathematicians and possibly even to some mathematicians who are interested in either complex analytic or algebraic geometric theories of moduli spaces. For example, who was the first to formulate the moduli problem precisely? What is the meaning of moduli spaces? Who was the first to give complex analytic and algebraic variety structures to Riemann's moduli space? Why did they study these problems? How was Riemann's moduli space first used? And why did Riemann choose and use the word moduli? Why did Teichmüller study Teichmüller space? What were Teichmüller's contributions to Riemann's moduli space? What were Teichmüller's contributions to algebraic geometry?
\end{abstract}

\section{Contents}

1 Introduction . . . . . . . . . . . . . . 46

2 Definitions . . . . . . . . . . . . . . 47

3 Riemann's Count of Moduli for Riemann Sur-

faces . . . . . . . . . . . . . . . . 49

* Department of Mathematics, University of Michigan, Ann Arbor, Mich., U.S.A.

E-mail: lji@umich.edu
4 Klein's Booklet on Riemann's Work and the Method of Continuity to Prove the Uniformization Theorem by Klein and Poincaré . 50

5 Teichmüller's Work on Moduli Space $\mathcal{M}_{g} \ldots 52$ 5.1 Teichmüller's Rigorous Formulation of and Results on Riemann's Moduli Space 52

5.2 Why Did Teichmüller Study Teichmüller Space and Riemann's Moduli Spaces? . . 57

6 Siegel Upper Half Space and Weil's Work on Weil-Petersson Metric . . . . . . . . . . . . 61

7 Grothendieck's Papers at Cartan Seminar . . . 62

8 Baily Compactification, Algebraic Structure on $\mathcal{M}_{g}$, and Torelli Morphism . . . . . . . . 64

9 Mumford's Work on $\mathcal{M}_{g}$ and Moduli Spaces . 67 10 Ahlfors and Bers' Works on Complex Analytic Geometry on Moduli Spaces . . . . . . . . 69 11 Meanings of Moduli in Moduli Spaces . . . . 70 Acknowledgments . . . . . . . . . . . . . 71 References . . . . . . . . . . . . . . . . . . . 71

\section{Introduction}

Mathematics is interesting and complicated. It also has interesting and complicated stories, which are often intriguing. Heroes can be mathematicians, or mathematical concepts and results. But interaction between them is probably more interesting.

In this article, we will tell the story of Riemann's moduli space. There are several reasons for choosing this topic. First, it had an honorable origin in the masterpiece "The theory of Abelian functions" by Riemann in 1857 [65, pp. 79-134]. Second, Riemann's moduli space of Riemann surfaces has been attracting more and more of people's attention, and research on it will probably continue for a long time 


\section{The Story of Riemann's Moduli Space}

\section{by Lizhen $\mathrm{Ji}^{*}$}

\begin{abstract}
Compact Riemann surfaces and projective algebraic curves over $\mathbb{C}$ are two realizations of the same class of objects. Their moduli space was first introduced by Riemann and is one of the central objects of the contemporary mathematics and mathematical physics. In this note, we explain the birth and evolution of Riemann's moduli space by emphasizing several points which might not be so well-known to the general mathematicians and possibly even to some mathematicians who are interested in either complex analytic or algebraic geometric theories of moduli spaces. For example, who was the first to formulate the moduli problem precisely? What is the meaning of moduli spaces? Who was the first to give complex analytic and algebraic variety structures to Riemann's moduli space? Why did they study these problems? How was Riemann's moduli space first used? And why did Riemann choose and use the word moduli? Why did Teichmüller study Teichmüller space? What were Teichmüller's contributions to Riemann's moduli space? What were Teichmüller's contributions to algebraic geometry?
\end{abstract}

\section{Contents}

1 Introduction . . . . . . . . . . . . . . 46

2 Definitions . . . . . . . . . . . . . . 47

3 Riemann's Count of Moduli for Riemann Sur-

faces . . . . . . . . . . . . . . . . 49

* Department of Mathematics, University of Michigan, Ann Arbor, Mich., U.S.A.

E-mail: lji@umich.edu
4 Klein's Booklet on Riemann's Work and the Method of Continuity to Prove the Uniformization Theorem by Klein and Poincaré . 50

5 Teichmüller's Work on Moduli Space $\mathcal{M}_{g} \ldots 52$ 5.1 Teichmüller's Rigorous Formulation of and Results on Riemann's Moduli Space 52

5.2 Why Did Teichmüller Study Teichmüller Space and Riemann's Moduli Spaces? . . 57

6 Siegel Upper Half Space and Weil's Work on Weil-Petersson Metric . . . . . . . . . . . . 61

7 Grothendieck's Papers at Cartan Seminar . . . 62

8 Baily Compactification, Algebraic Structure on $\mathcal{M}_{g}$, and Torelli Morphism . . . . . . . . 64

9 Mumford's Work on $\mathcal{M}_{g}$ and Moduli Spaces . 67 10 Ahlfors and Bers' Works on Complex Analytic Geometry on Moduli Spaces . . . . . . . . 69 11 Meanings of Moduli in Moduli Spaces . . . . 70 Acknowledgments . . . . . . . . . . . . . 71 References . . . . . . . . . . . . . . . . . . . 71

\section{Introduction}

Mathematics is interesting and complicated. It also has interesting and complicated stories, which are often intriguing. Heroes can be mathematicians, or mathematical concepts and results. But interaction between them is probably more interesting.

In this article, we will tell the story of Riemann's moduli space. There are several reasons for choosing this topic. First, it had an honorable origin in the masterpiece "The theory of Abelian functions" by Riemann in 1857 [65, pp. 79-134]. Second, Riemann's moduli space of Riemann surfaces has been attracting more and more of people's attention, and research on it will probably continue for a long time 


\section{The Story of Riemann's Moduli Space}

\section{by Lizhen $\mathrm{Ji}^{*}$}

\begin{abstract}
Compact Riemann surfaces and projective algebraic curves over $\mathbb{C}$ are two realizations of the same class of objects. Their moduli space was first introduced by Riemann and is one of the central objects of the contemporary mathematics and mathematical physics. In this note, we explain the birth and evolution of Riemann's moduli space by emphasizing several points which might not be so well-known to the general mathematicians and possibly even to some mathematicians who are interested in either complex analytic or algebraic geometric theories of moduli spaces. For example, who was the first to formulate the moduli problem precisely? What is the meaning of moduli spaces? Who was the first to give complex analytic and algebraic variety structures to Riemann's moduli space? Why did they study these problems? How was Riemann's moduli space first used? And why did Riemann choose and use the word moduli? Why did Teichmüller study Teichmüller space? What were Teichmüller's contributions to Riemann's moduli space? What were Teichmüller's contributions to algebraic geometry?
\end{abstract}

\section{Contents}

1 Introduction . . . . . . . . . . . . . . 46

2 Definitions . . . . . . . . . . . . . . 47

3 Riemann's Count of Moduli for Riemann Sur-

faces . . . . . . . . . . . . . . . . 49

* Department of Mathematics, University of Michigan, Ann Arbor, Mich., U.S.A.

E-mail: lji@umich.edu
4 Klein's Booklet on Riemann's Work and the Method of Continuity to Prove the Uniformization Theorem by Klein and Poincaré . 50

5 Teichmüller's Work on Moduli Space $\mathcal{M}_{g} \ldots 52$ 5.1 Teichmüller's Rigorous Formulation of and Results on Riemann's Moduli Space 52

5.2 Why Did Teichmüller Study Teichmüller Space and Riemann's Moduli Spaces? . . 57

6 Siegel Upper Half Space and Weil's Work on Weil-Petersson Metric . . . . . . . . . . . . 61

7 Grothendieck's Papers at Cartan Seminar . . . 62

8 Baily Compactification, Algebraic Structure on $\mathcal{M}_{g}$, and Torelli Morphism . . . . . . . . 64

9 Mumford's Work on $\mathcal{M}_{g}$ and Moduli Spaces . 67 10 Ahlfors and Bers' Works on Complex Analytic Geometry on Moduli Spaces . . . . . . . . 69 11 Meanings of Moduli in Moduli Spaces . . . . 70 Acknowledgments . . . . . . . . . . . . . 71 References . . . . . . . . . . . . . . . . . . . 71

\section{Introduction}

Mathematics is interesting and complicated. It also has interesting and complicated stories, which are often intriguing. Heroes can be mathematicians, or mathematical concepts and results. But interaction between them is probably more interesting.

In this article, we will tell the story of Riemann's moduli space. There are several reasons for choosing this topic. First, it had an honorable origin in the masterpiece "The theory of Abelian functions" by Riemann in 1857 [65, pp. 79-134]. Second, Riemann's moduli space of Riemann surfaces has been attracting more and more of people's attention, and research on it will probably continue for a long time 


\section{The Story of Riemann's Moduli Space}

\section{by Lizhen $\mathrm{Ji}^{*}$}

\begin{abstract}
Compact Riemann surfaces and projective algebraic curves over $\mathbb{C}$ are two realizations of the same class of objects. Their moduli space was first introduced by Riemann and is one of the central objects of the contemporary mathematics and mathematical physics. In this note, we explain the birth and evolution of Riemann's moduli space by emphasizing several points which might not be so well-known to the general mathematicians and possibly even to some mathematicians who are interested in either complex analytic or algebraic geometric theories of moduli spaces. For example, who was the first to formulate the moduli problem precisely? What is the meaning of moduli spaces? Who was the first to give complex analytic and algebraic variety structures to Riemann's moduli space? Why did they study these problems? How was Riemann's moduli space first used? And why did Riemann choose and use the word moduli? Why did Teichmüller study Teichmüller space? What were Teichmüller's contributions to Riemann's moduli space? What were Teichmüller's contributions to algebraic geometry?
\end{abstract}

\section{Contents}

1 Introduction . . . . . . . . . . . . . . 46

2 Definitions . . . . . . . . . . . . . . 47

3 Riemann's Count of Moduli for Riemann Sur-

faces . . . . . . . . . . . . . . . . 49

* Department of Mathematics, University of Michigan, Ann Arbor, Mich., U.S.A.

E-mail: lji@umich.edu
4 Klein's Booklet on Riemann's Work and the Method of Continuity to Prove the Uniformization Theorem by Klein and Poincaré . 50

5 Teichmüller's Work on Moduli Space $\mathcal{M}_{g} \ldots 52$ 5.1 Teichmüller's Rigorous Formulation of and Results on Riemann's Moduli Space 52

5.2 Why Did Teichmüller Study Teichmüller Space and Riemann's Moduli Spaces? . . 57

6 Siegel Upper Half Space and Weil's Work on Weil-Petersson Metric . . . . . . . . . . . . 61

7 Grothendieck's Papers at Cartan Seminar . . . 62

8 Baily Compactification, Algebraic Structure on $\mathcal{M}_{g}$, and Torelli Morphism . . . . . . . . 64

9 Mumford's Work on $\mathcal{M}_{g}$ and Moduli Spaces . 67 10 Ahlfors and Bers' Works on Complex Analytic Geometry on Moduli Spaces . . . . . . . . 69 11 Meanings of Moduli in Moduli Spaces . . . . 70 Acknowledgments . . . . . . . . . . . . . 71 References . . . . . . . . . . . . . . . . . . . 71

\section{Introduction}

Mathematics is interesting and complicated. It also has interesting and complicated stories, which are often intriguing. Heroes can be mathematicians, or mathematical concepts and results. But interaction between them is probably more interesting.

In this article, we will tell the story of Riemann's moduli space. There are several reasons for choosing this topic. First, it had an honorable origin in the masterpiece "The theory of Abelian functions" by Riemann in 1857 [65, pp. 79-134]. Second, Riemann's moduli space of Riemann surfaces has been attracting more and more of people's attention, and research on it will probably continue for a long time 


\section{The Story of Riemann's Moduli Space}

\section{by Lizhen $\mathrm{Ji}^{*}$}

\begin{abstract}
Compact Riemann surfaces and projective algebraic curves over $\mathbb{C}$ are two realizations of the same class of objects. Their moduli space was first introduced by Riemann and is one of the central objects of the contemporary mathematics and mathematical physics. In this note, we explain the birth and evolution of Riemann's moduli space by emphasizing several points which might not be so well-known to the general mathematicians and possibly even to some mathematicians who are interested in either complex analytic or algebraic geometric theories of moduli spaces. For example, who was the first to formulate the moduli problem precisely? What is the meaning of moduli spaces? Who was the first to give complex analytic and algebraic variety structures to Riemann's moduli space? Why did they study these problems? How was Riemann's moduli space first used? And why did Riemann choose and use the word moduli? Why did Teichmüller study Teichmüller space? What were Teichmüller's contributions to Riemann's moduli space? What were Teichmüller's contributions to algebraic geometry?
\end{abstract}

\section{Contents}

1 Introduction . . . . . . . . . . . . . . 46

2 Definitions . . . . . . . . . . . . . . 47

3 Riemann's Count of Moduli for Riemann Sur-

faces . . . . . . . . . . . . . . . . 49

* Department of Mathematics, University of Michigan, Ann Arbor, Mich., U.S.A.

E-mail: lji@umich.edu
4 Klein's Booklet on Riemann's Work and the Method of Continuity to Prove the Uniformization Theorem by Klein and Poincaré . 50

5 Teichmüller's Work on Moduli Space $\mathcal{M}_{g} \ldots 52$ 5.1 Teichmüller's Rigorous Formulation of and Results on Riemann's Moduli Space 52

5.2 Why Did Teichmüller Study Teichmüller Space and Riemann's Moduli Spaces? . . 57

6 Siegel Upper Half Space and Weil's Work on Weil-Petersson Metric . . . . . . . . . . . . 61

7 Grothendieck's Papers at Cartan Seminar . . . 62

8 Baily Compactification, Algebraic Structure on $\mathcal{M}_{g}$, and Torelli Morphism . . . . . . . . 64

9 Mumford's Work on $\mathcal{M}_{g}$ and Moduli Spaces . 67 10 Ahlfors and Bers' Works on Complex Analytic Geometry on Moduli Spaces . . . . . . . . 69 11 Meanings of Moduli in Moduli Spaces . . . . 70 Acknowledgments . . . . . . . . . . . . . 71 References . . . . . . . . . . . . . . . . . . . 71

\section{Introduction}

Mathematics is interesting and complicated. It also has interesting and complicated stories, which are often intriguing. Heroes can be mathematicians, or mathematical concepts and results. But interaction between them is probably more interesting.

In this article, we will tell the story of Riemann's moduli space. There are several reasons for choosing this topic. First, it had an honorable origin in the masterpiece "The theory of Abelian functions" by Riemann in 1857 [65, pp. 79-134]. Second, Riemann's moduli space of Riemann surfaces has been attracting more and more of people's attention, and research on it will probably continue for a long time 


\section{The Story of Riemann's Moduli Space}

\section{by Lizhen $\mathrm{Ji}^{*}$}

\begin{abstract}
Compact Riemann surfaces and projective algebraic curves over $\mathbb{C}$ are two realizations of the same class of objects. Their moduli space was first introduced by Riemann and is one of the central objects of the contemporary mathematics and mathematical physics. In this note, we explain the birth and evolution of Riemann's moduli space by emphasizing several points which might not be so well-known to the general mathematicians and possibly even to some mathematicians who are interested in either complex analytic or algebraic geometric theories of moduli spaces. For example, who was the first to formulate the moduli problem precisely? What is the meaning of moduli spaces? Who was the first to give complex analytic and algebraic variety structures to Riemann's moduli space? Why did they study these problems? How was Riemann's moduli space first used? And why did Riemann choose and use the word moduli? Why did Teichmüller study Teichmüller space? What were Teichmüller's contributions to Riemann's moduli space? What were Teichmüller's contributions to algebraic geometry?
\end{abstract}

\section{Contents}

1 Introduction . . . . . . . . . . . . . . 46

2 Definitions . . . . . . . . . . . . . . 47

3 Riemann's Count of Moduli for Riemann Sur-

faces . . . . . . . . . . . . . . . . 49

* Department of Mathematics, University of Michigan, Ann Arbor, Mich., U.S.A.

E-mail: lji@umich.edu
4 Klein's Booklet on Riemann's Work and the Method of Continuity to Prove the Uniformization Theorem by Klein and Poincaré . 50

5 Teichmüller's Work on Moduli Space $\mathcal{M}_{g} \ldots 52$ 5.1 Teichmüller's Rigorous Formulation of and Results on Riemann's Moduli Space 52

5.2 Why Did Teichmüller Study Teichmüller Space and Riemann's Moduli Spaces? . . 57

6 Siegel Upper Half Space and Weil's Work on Weil-Petersson Metric . . . . . . . . . . . . 61

7 Grothendieck's Papers at Cartan Seminar . . . 62

8 Baily Compactification, Algebraic Structure on $\mathcal{M}_{g}$, and Torelli Morphism . . . . . . . . 64

9 Mumford's Work on $\mathcal{M}_{g}$ and Moduli Spaces . 67 10 Ahlfors and Bers' Works on Complex Analytic Geometry on Moduli Spaces . . . . . . . . 69 11 Meanings of Moduli in Moduli Spaces . . . . 70 Acknowledgments . . . . . . . . . . . . . 71 References . . . . . . . . . . . . . . . . . . . 71

\section{Introduction}

Mathematics is interesting and complicated. It also has interesting and complicated stories, which are often intriguing. Heroes can be mathematicians, or mathematical concepts and results. But interaction between them is probably more interesting.

In this article, we will tell the story of Riemann's moduli space. There are several reasons for choosing this topic. First, it had an honorable origin in the masterpiece "The theory of Abelian functions" by Riemann in 1857 [65, pp. 79-134]. Second, Riemann's moduli space of Riemann surfaces has been attracting more and more of people's attention, and research on it will probably continue for a long time 


\section{The Story of Riemann's Moduli Space}

\section{by Lizhen $\mathrm{Ji}^{*}$}

\begin{abstract}
Compact Riemann surfaces and projective algebraic curves over $\mathbb{C}$ are two realizations of the same class of objects. Their moduli space was first introduced by Riemann and is one of the central objects of the contemporary mathematics and mathematical physics. In this note, we explain the birth and evolution of Riemann's moduli space by emphasizing several points which might not be so well-known to the general mathematicians and possibly even to some mathematicians who are interested in either complex analytic or algebraic geometric theories of moduli spaces. For example, who was the first to formulate the moduli problem precisely? What is the meaning of moduli spaces? Who was the first to give complex analytic and algebraic variety structures to Riemann's moduli space? Why did they study these problems? How was Riemann's moduli space first used? And why did Riemann choose and use the word moduli? Why did Teichmüller study Teichmüller space? What were Teichmüller's contributions to Riemann's moduli space? What were Teichmüller's contributions to algebraic geometry?
\end{abstract}

\section{Contents}

1 Introduction . . . . . . . . . . . . . . 46

2 Definitions . . . . . . . . . . . . . . 47

3 Riemann's Count of Moduli for Riemann Sur-

faces . . . . . . . . . . . . . . . . 49

* Department of Mathematics, University of Michigan, Ann Arbor, Mich., U.S.A.

E-mail: lji@umich.edu
4 Klein's Booklet on Riemann's Work and the Method of Continuity to Prove the Uniformization Theorem by Klein and Poincaré . 50

5 Teichmüller's Work on Moduli Space $\mathcal{M}_{g} \ldots 52$ 5.1 Teichmüller's Rigorous Formulation of and Results on Riemann's Moduli Space 52

5.2 Why Did Teichmüller Study Teichmüller Space and Riemann's Moduli Spaces? . . 57

6 Siegel Upper Half Space and Weil's Work on Weil-Petersson Metric . . . . . . . . . . . . 61

7 Grothendieck's Papers at Cartan Seminar . . . 62

8 Baily Compactification, Algebraic Structure on $\mathcal{M}_{g}$, and Torelli Morphism . . . . . . . . 64

9 Mumford's Work on $\mathcal{M}_{g}$ and Moduli Spaces . 67 10 Ahlfors and Bers' Works on Complex Analytic Geometry on Moduli Spaces . . . . . . . . 69 11 Meanings of Moduli in Moduli Spaces . . . . 70 Acknowledgments . . . . . . . . . . . . . 71 References . . . . . . . . . . . . . . . . . . . 71

\section{Introduction}

Mathematics is interesting and complicated. It also has interesting and complicated stories, which are often intriguing. Heroes can be mathematicians, or mathematical concepts and results. But interaction between them is probably more interesting.

In this article, we will tell the story of Riemann's moduli space. There are several reasons for choosing this topic. First, it had an honorable origin in the masterpiece "The theory of Abelian functions" by Riemann in 1857 [65, pp. 79-134]. Second, Riemann's moduli space of Riemann surfaces has been attracting more and more of people's attention, and research on it will probably continue for a long time 


\section{The Story of Riemann's Moduli Space}

\section{by Lizhen $\mathrm{Ji}^{*}$}

\begin{abstract}
Compact Riemann surfaces and projective algebraic curves over $\mathbb{C}$ are two realizations of the same class of objects. Their moduli space was first introduced by Riemann and is one of the central objects of the contemporary mathematics and mathematical physics. In this note, we explain the birth and evolution of Riemann's moduli space by emphasizing several points which might not be so well-known to the general mathematicians and possibly even to some mathematicians who are interested in either complex analytic or algebraic geometric theories of moduli spaces. For example, who was the first to formulate the moduli problem precisely? What is the meaning of moduli spaces? Who was the first to give complex analytic and algebraic variety structures to Riemann's moduli space? Why did they study these problems? How was Riemann's moduli space first used? And why did Riemann choose and use the word moduli? Why did Teichmüller study Teichmüller space? What were Teichmüller's contributions to Riemann's moduli space? What were Teichmüller's contributions to algebraic geometry?
\end{abstract}

\section{Contents}

1 Introduction . . . . . . . . . . . . . . 46

2 Definitions . . . . . . . . . . . . . . 47

3 Riemann's Count of Moduli for Riemann Sur-

faces . . . . . . . . . . . . . . . . 49

* Department of Mathematics, University of Michigan, Ann Arbor, Mich., U.S.A.

E-mail: lji@umich.edu
4 Klein's Booklet on Riemann's Work and the Method of Continuity to Prove the Uniformization Theorem by Klein and Poincaré . 50

5 Teichmüller's Work on Moduli Space $\mathcal{M}_{g} \ldots 52$ 5.1 Teichmüller's Rigorous Formulation of and Results on Riemann's Moduli Space 52

5.2 Why Did Teichmüller Study Teichmüller Space and Riemann's Moduli Spaces? . . 57

6 Siegel Upper Half Space and Weil's Work on Weil-Petersson Metric . . . . . . . . . . . . 61

7 Grothendieck's Papers at Cartan Seminar . . . 62

8 Baily Compactification, Algebraic Structure on $\mathcal{M}_{g}$, and Torelli Morphism . . . . . . . . 64

9 Mumford's Work on $\mathcal{M}_{g}$ and Moduli Spaces . 67 10 Ahlfors and Bers' Works on Complex Analytic Geometry on Moduli Spaces . . . . . . . . 69 11 Meanings of Moduli in Moduli Spaces . . . . 70 Acknowledgments . . . . . . . . . . . . . 71 References . . . . . . . . . . . . . . . . . . . 71

\section{Introduction}

Mathematics is interesting and complicated. It also has interesting and complicated stories, which are often intriguing. Heroes can be mathematicians, or mathematical concepts and results. But interaction between them is probably more interesting.

In this article, we will tell the story of Riemann's moduli space. There are several reasons for choosing this topic. First, it had an honorable origin in the masterpiece "The theory of Abelian functions" by Riemann in 1857 [65, pp. 79-134]. Second, Riemann's moduli space of Riemann surfaces has been attracting more and more of people's attention, and research on it will probably continue for a long time 


\section{The Story of Riemann's Moduli Space}

\section{by Lizhen $\mathrm{Ji}^{*}$}

\begin{abstract}
Compact Riemann surfaces and projective algebraic curves over $\mathbb{C}$ are two realizations of the same class of objects. Their moduli space was first introduced by Riemann and is one of the central objects of the contemporary mathematics and mathematical physics. In this note, we explain the birth and evolution of Riemann's moduli space by emphasizing several points which might not be so well-known to the general mathematicians and possibly even to some mathematicians who are interested in either complex analytic or algebraic geometric theories of moduli spaces. For example, who was the first to formulate the moduli problem precisely? What is the meaning of moduli spaces? Who was the first to give complex analytic and algebraic variety structures to Riemann's moduli space? Why did they study these problems? How was Riemann's moduli space first used? And why did Riemann choose and use the word moduli? Why did Teichmüller study Teichmüller space? What were Teichmüller's contributions to Riemann's moduli space? What were Teichmüller's contributions to algebraic geometry?
\end{abstract}

\section{Contents}

1 Introduction . . . . . . . . . . . . . . 46

2 Definitions . . . . . . . . . . . . . . 47

3 Riemann's Count of Moduli for Riemann Sur-

faces . . . . . . . . . . . . . . . . 49

* Department of Mathematics, University of Michigan, Ann Arbor, Mich., U.S.A.

E-mail: lji@umich.edu
4 Klein's Booklet on Riemann's Work and the Method of Continuity to Prove the Uniformization Theorem by Klein and Poincaré . 50

5 Teichmüller's Work on Moduli Space $\mathcal{M}_{g} \ldots 52$ 5.1 Teichmüller's Rigorous Formulation of and Results on Riemann's Moduli Space 52

5.2 Why Did Teichmüller Study Teichmüller Space and Riemann's Moduli Spaces? . . 57

6 Siegel Upper Half Space and Weil's Work on Weil-Petersson Metric . . . . . . . . . . . . 61

7 Grothendieck's Papers at Cartan Seminar . . . 62

8 Baily Compactification, Algebraic Structure on $\mathcal{M}_{g}$, and Torelli Morphism . . . . . . . . 64

9 Mumford's Work on $\mathcal{M}_{g}$ and Moduli Spaces . 67 10 Ahlfors and Bers' Works on Complex Analytic Geometry on Moduli Spaces . . . . . . . . 69 11 Meanings of Moduli in Moduli Spaces . . . . 70 Acknowledgments . . . . . . . . . . . . . 71 References . . . . . . . . . . . . . . . . . . . 71

\section{Introduction}

Mathematics is interesting and complicated. It also has interesting and complicated stories, which are often intriguing. Heroes can be mathematicians, or mathematical concepts and results. But interaction between them is probably more interesting.

In this article, we will tell the story of Riemann's moduli space. There are several reasons for choosing this topic. First, it had an honorable origin in the masterpiece "The theory of Abelian functions" by Riemann in 1857 [65, pp. 79-134]. Second, Riemann's moduli space of Riemann surfaces has been attracting more and more of people's attention, and research on it will probably continue for a long time 


\section{The Story of Riemann's Moduli Space}

\section{by Lizhen $\mathrm{Ji}^{*}$}

\begin{abstract}
Compact Riemann surfaces and projective algebraic curves over $\mathbb{C}$ are two realizations of the same class of objects. Their moduli space was first introduced by Riemann and is one of the central objects of the contemporary mathematics and mathematical physics. In this note, we explain the birth and evolution of Riemann's moduli space by emphasizing several points which might not be so well-known to the general mathematicians and possibly even to some mathematicians who are interested in either complex analytic or algebraic geometric theories of moduli spaces. For example, who was the first to formulate the moduli problem precisely? What is the meaning of moduli spaces? Who was the first to give complex analytic and algebraic variety structures to Riemann's moduli space? Why did they study these problems? How was Riemann's moduli space first used? And why did Riemann choose and use the word moduli? Why did Teichmüller study Teichmüller space? What were Teichmüller's contributions to Riemann's moduli space? What were Teichmüller's contributions to algebraic geometry?
\end{abstract}

\section{Contents}

1 Introduction . . . . . . . . . . . . . . 46

2 Definitions . . . . . . . . . . . . . . 47

3 Riemann's Count of Moduli for Riemann Sur-

faces . . . . . . . . . . . . . . . . 49

* Department of Mathematics, University of Michigan, Ann Arbor, Mich., U.S.A.

E-mail: lji@umich.edu
4 Klein's Booklet on Riemann's Work and the Method of Continuity to Prove the Uniformization Theorem by Klein and Poincaré . 50

5 Teichmüller's Work on Moduli Space $\mathcal{M}_{g} \ldots 52$ 5.1 Teichmüller's Rigorous Formulation of and Results on Riemann's Moduli Space 52

5.2 Why Did Teichmüller Study Teichmüller Space and Riemann's Moduli Spaces? . . 57

6 Siegel Upper Half Space and Weil's Work on Weil-Petersson Metric . . . . . . . . . . . . 61

7 Grothendieck's Papers at Cartan Seminar . . . 62

8 Baily Compactification, Algebraic Structure on $\mathcal{M}_{g}$, and Torelli Morphism . . . . . . . . 64

9 Mumford's Work on $\mathcal{M}_{g}$ and Moduli Spaces . 67 10 Ahlfors and Bers' Works on Complex Analytic Geometry on Moduli Spaces . . . . . . . . 69 11 Meanings of Moduli in Moduli Spaces . . . . 70 Acknowledgments . . . . . . . . . . . . . 71 References . . . . . . . . . . . . . . . . . . . 71

\section{Introduction}

Mathematics is interesting and complicated. It also has interesting and complicated stories, which are often intriguing. Heroes can be mathematicians, or mathematical concepts and results. But interaction between them is probably more interesting.

In this article, we will tell the story of Riemann's moduli space. There are several reasons for choosing this topic. First, it had an honorable origin in the masterpiece "The theory of Abelian functions" by Riemann in 1857 [65, pp. 79-134]. Second, Riemann's moduli space of Riemann surfaces has been attracting more and more of people's attention, and research on it will probably continue for a long time 


\section{The Story of Riemann's Moduli Space}

\section{by Lizhen $\mathrm{Ji}^{*}$}

\begin{abstract}
Compact Riemann surfaces and projective algebraic curves over $\mathbb{C}$ are two realizations of the same class of objects. Their moduli space was first introduced by Riemann and is one of the central objects of the contemporary mathematics and mathematical physics. In this note, we explain the birth and evolution of Riemann's moduli space by emphasizing several points which might not be so well-known to the general mathematicians and possibly even to some mathematicians who are interested in either complex analytic or algebraic geometric theories of moduli spaces. For example, who was the first to formulate the moduli problem precisely? What is the meaning of moduli spaces? Who was the first to give complex analytic and algebraic variety structures to Riemann's moduli space? Why did they study these problems? How was Riemann's moduli space first used? And why did Riemann choose and use the word moduli? Why did Teichmüller study Teichmüller space? What were Teichmüller's contributions to Riemann's moduli space? What were Teichmüller's contributions to algebraic geometry?
\end{abstract}

\section{Contents}

1 Introduction . . . . . . . . . . . . . . 46

2 Definitions . . . . . . . . . . . . . . 47

3 Riemann's Count of Moduli for Riemann Sur-

faces . . . . . . . . . . . . . . . . 49

* Department of Mathematics, University of Michigan, Ann Arbor, Mich., U.S.A.

E-mail: lji@umich.edu
4 Klein's Booklet on Riemann's Work and the Method of Continuity to Prove the Uniformization Theorem by Klein and Poincaré . 50

5 Teichmüller's Work on Moduli Space $\mathcal{M}_{g} \ldots 52$ 5.1 Teichmüller's Rigorous Formulation of and Results on Riemann's Moduli Space 52

5.2 Why Did Teichmüller Study Teichmüller Space and Riemann's Moduli Spaces? . . 57

6 Siegel Upper Half Space and Weil's Work on Weil-Petersson Metric . . . . . . . . . . . . 61

7 Grothendieck's Papers at Cartan Seminar . . . 62

8 Baily Compactification, Algebraic Structure on $\mathcal{M}_{g}$, and Torelli Morphism . . . . . . . . 64

9 Mumford's Work on $\mathcal{M}_{g}$ and Moduli Spaces . 67 10 Ahlfors and Bers' Works on Complex Analytic Geometry on Moduli Spaces . . . . . . . . 69 11 Meanings of Moduli in Moduli Spaces . . . . 70 Acknowledgments . . . . . . . . . . . . . 71 References . . . . . . . . . . . . . . . . . . . 71

\section{Introduction}

Mathematics is interesting and complicated. It also has interesting and complicated stories, which are often intriguing. Heroes can be mathematicians, or mathematical concepts and results. But interaction between them is probably more interesting.

In this article, we will tell the story of Riemann's moduli space. There are several reasons for choosing this topic. First, it had an honorable origin in the masterpiece "The theory of Abelian functions" by Riemann in 1857 [65, pp. 79-134]. Second, Riemann's moduli space of Riemann surfaces has been attracting more and more of people's attention, and research on it will probably continue for a long time 


\section{The Story of Riemann's Moduli Space}

\section{by Lizhen $\mathrm{Ji}^{*}$}

\begin{abstract}
Compact Riemann surfaces and projective algebraic curves over $\mathbb{C}$ are two realizations of the same class of objects. Their moduli space was first introduced by Riemann and is one of the central objects of the contemporary mathematics and mathematical physics. In this note, we explain the birth and evolution of Riemann's moduli space by emphasizing several points which might not be so well-known to the general mathematicians and possibly even to some mathematicians who are interested in either complex analytic or algebraic geometric theories of moduli spaces. For example, who was the first to formulate the moduli problem precisely? What is the meaning of moduli spaces? Who was the first to give complex analytic and algebraic variety structures to Riemann's moduli space? Why did they study these problems? How was Riemann's moduli space first used? And why did Riemann choose and use the word moduli? Why did Teichmüller study Teichmüller space? What were Teichmüller's contributions to Riemann's moduli space? What were Teichmüller's contributions to algebraic geometry?
\end{abstract}

\section{Contents}

1 Introduction . . . . . . . . . . . . . . 46

2 Definitions . . . . . . . . . . . . . . 47

3 Riemann's Count of Moduli for Riemann Sur-

faces . . . . . . . . . . . . . . . . 49

* Department of Mathematics, University of Michigan, Ann Arbor, Mich., U.S.A.

E-mail: lji@umich.edu
4 Klein's Booklet on Riemann's Work and the Method of Continuity to Prove the Uniformization Theorem by Klein and Poincaré . 50

5 Teichmüller's Work on Moduli Space $\mathcal{M}_{g} \ldots 52$ 5.1 Teichmüller's Rigorous Formulation of and Results on Riemann's Moduli Space 52

5.2 Why Did Teichmüller Study Teichmüller Space and Riemann's Moduli Spaces? . . 57

6 Siegel Upper Half Space and Weil's Work on Weil-Petersson Metric . . . . . . . . . . . . 61

7 Grothendieck's Papers at Cartan Seminar . . . 62

8 Baily Compactification, Algebraic Structure on $\mathcal{M}_{g}$, and Torelli Morphism . . . . . . . . 64

9 Mumford's Work on $\mathcal{M}_{g}$ and Moduli Spaces . 67 10 Ahlfors and Bers' Works on Complex Analytic Geometry on Moduli Spaces . . . . . . . . 69 11 Meanings of Moduli in Moduli Spaces . . . . 70 Acknowledgments . . . . . . . . . . . . . 71 References . . . . . . . . . . . . . . . . . . . 71

\section{Introduction}

Mathematics is interesting and complicated. It also has interesting and complicated stories, which are often intriguing. Heroes can be mathematicians, or mathematical concepts and results. But interaction between them is probably more interesting.

In this article, we will tell the story of Riemann's moduli space. There are several reasons for choosing this topic. First, it had an honorable origin in the masterpiece "The theory of Abelian functions" by Riemann in 1857 [65, pp. 79-134]. Second, Riemann's moduli space of Riemann surfaces has been attracting more and more of people's attention, and research on it will probably continue for a long time 


\section{The Story of Riemann's Moduli Space}

\section{by Lizhen $\mathrm{Ji}^{*}$}

\begin{abstract}
Compact Riemann surfaces and projective algebraic curves over $\mathbb{C}$ are two realizations of the same class of objects. Their moduli space was first introduced by Riemann and is one of the central objects of the contemporary mathematics and mathematical physics. In this note, we explain the birth and evolution of Riemann's moduli space by emphasizing several points which might not be so well-known to the general mathematicians and possibly even to some mathematicians who are interested in either complex analytic or algebraic geometric theories of moduli spaces. For example, who was the first to formulate the moduli problem precisely? What is the meaning of moduli spaces? Who was the first to give complex analytic and algebraic variety structures to Riemann's moduli space? Why did they study these problems? How was Riemann's moduli space first used? And why did Riemann choose and use the word moduli? Why did Teichmüller study Teichmüller space? What were Teichmüller's contributions to Riemann's moduli space? What were Teichmüller's contributions to algebraic geometry?
\end{abstract}

\section{Contents}

1 Introduction . . . . . . . . . . . . . . 46

2 Definitions . . . . . . . . . . . . . . 47

3 Riemann's Count of Moduli for Riemann Sur-

faces . . . . . . . . . . . . . . . . 49

* Department of Mathematics, University of Michigan, Ann Arbor, Mich., U.S.A.

E-mail: lji@umich.edu
4 Klein's Booklet on Riemann's Work and the Method of Continuity to Prove the Uniformization Theorem by Klein and Poincaré . 50

5 Teichmüller's Work on Moduli Space $\mathcal{M}_{g} \ldots 52$ 5.1 Teichmüller's Rigorous Formulation of and Results on Riemann's Moduli Space 52

5.2 Why Did Teichmüller Study Teichmüller Space and Riemann's Moduli Spaces? . . 57

6 Siegel Upper Half Space and Weil's Work on Weil-Petersson Metric . . . . . . . . . . . . 61

7 Grothendieck's Papers at Cartan Seminar . . . 62

8 Baily Compactification, Algebraic Structure on $\mathcal{M}_{g}$, and Torelli Morphism . . . . . . . . 64

9 Mumford's Work on $\mathcal{M}_{g}$ and Moduli Spaces . 67 10 Ahlfors and Bers' Works on Complex Analytic Geometry on Moduli Spaces . . . . . . . . 69 11 Meanings of Moduli in Moduli Spaces . . . . 70 Acknowledgments . . . . . . . . . . . . . 71 References . . . . . . . . . . . . . . . . . . . 71

\section{Introduction}

Mathematics is interesting and complicated. It also has interesting and complicated stories, which are often intriguing. Heroes can be mathematicians, or mathematical concepts and results. But interaction between them is probably more interesting.

In this article, we will tell the story of Riemann's moduli space. There are several reasons for choosing this topic. First, it had an honorable origin in the masterpiece "The theory of Abelian functions" by Riemann in 1857 [65, pp. 79-134]. Second, Riemann's moduli space of Riemann surfaces has been attracting more and more of people's attention, and research on it will probably continue for a long time 


\section{The Story of Riemann's Moduli Space}

\section{by Lizhen $\mathrm{Ji}^{*}$}

\begin{abstract}
Compact Riemann surfaces and projective algebraic curves over $\mathbb{C}$ are two realizations of the same class of objects. Their moduli space was first introduced by Riemann and is one of the central objects of the contemporary mathematics and mathematical physics. In this note, we explain the birth and evolution of Riemann's moduli space by emphasizing several points which might not be so well-known to the general mathematicians and possibly even to some mathematicians who are interested in either complex analytic or algebraic geometric theories of moduli spaces. For example, who was the first to formulate the moduli problem precisely? What is the meaning of moduli spaces? Who was the first to give complex analytic and algebraic variety structures to Riemann's moduli space? Why did they study these problems? How was Riemann's moduli space first used? And why did Riemann choose and use the word moduli? Why did Teichmüller study Teichmüller space? What were Teichmüller's contributions to Riemann's moduli space? What were Teichmüller's contributions to algebraic geometry?
\end{abstract}

\section{Contents}

1 Introduction . . . . . . . . . . . . . . 46

2 Definitions . . . . . . . . . . . . . . 47

3 Riemann's Count of Moduli for Riemann Sur-

faces . . . . . . . . . . . . . . . . 49

* Department of Mathematics, University of Michigan, Ann Arbor, Mich., U.S.A.

E-mail: lji@umich.edu
4 Klein's Booklet on Riemann's Work and the Method of Continuity to Prove the Uniformization Theorem by Klein and Poincaré . 50

5 Teichmüller's Work on Moduli Space $\mathcal{M}_{g} \ldots 52$ 5.1 Teichmüller's Rigorous Formulation of and Results on Riemann's Moduli Space 52

5.2 Why Did Teichmüller Study Teichmüller Space and Riemann's Moduli Spaces? . . 57

6 Siegel Upper Half Space and Weil's Work on Weil-Petersson Metric . . . . . . . . . . . . 61

7 Grothendieck's Papers at Cartan Seminar . . . 62

8 Baily Compactification, Algebraic Structure on $\mathcal{M}_{g}$, and Torelli Morphism . . . . . . . . 64

9 Mumford's Work on $\mathcal{M}_{g}$ and Moduli Spaces . 67 10 Ahlfors and Bers' Works on Complex Analytic Geometry on Moduli Spaces . . . . . . . . 69 11 Meanings of Moduli in Moduli Spaces . . . . 70 Acknowledgments . . . . . . . . . . . . . 71 References . . . . . . . . . . . . . . . . . . . 71

\section{Introduction}

Mathematics is interesting and complicated. It also has interesting and complicated stories, which are often intriguing. Heroes can be mathematicians, or mathematical concepts and results. But interaction between them is probably more interesting.

In this article, we will tell the story of Riemann's moduli space. There are several reasons for choosing this topic. First, it had an honorable origin in the masterpiece "The theory of Abelian functions" by Riemann in 1857 [65, pp. 79-134]. Second, Riemann's moduli space of Riemann surfaces has been attracting more and more of people's attention, and research on it will probably continue for a long time 


\section{The Story of Riemann's Moduli Space}

\section{by Lizhen $\mathrm{Ji}^{*}$}

\begin{abstract}
Compact Riemann surfaces and projective algebraic curves over $\mathbb{C}$ are two realizations of the same class of objects. Their moduli space was first introduced by Riemann and is one of the central objects of the contemporary mathematics and mathematical physics. In this note, we explain the birth and evolution of Riemann's moduli space by emphasizing several points which might not be so well-known to the general mathematicians and possibly even to some mathematicians who are interested in either complex analytic or algebraic geometric theories of moduli spaces. For example, who was the first to formulate the moduli problem precisely? What is the meaning of moduli spaces? Who was the first to give complex analytic and algebraic variety structures to Riemann's moduli space? Why did they study these problems? How was Riemann's moduli space first used? And why did Riemann choose and use the word moduli? Why did Teichmüller study Teichmüller space? What were Teichmüller's contributions to Riemann's moduli space? What were Teichmüller's contributions to algebraic geometry?
\end{abstract}

\section{Contents}

1 Introduction . . . . . . . . . . . . . . 46

2 Definitions . . . . . . . . . . . . . . 47

3 Riemann's Count of Moduli for Riemann Sur-

faces . . . . . . . . . . . . . . . . 49

* Department of Mathematics, University of Michigan, Ann Arbor, Mich., U.S.A.

E-mail: lji@umich.edu
4 Klein's Booklet on Riemann's Work and the Method of Continuity to Prove the Uniformization Theorem by Klein and Poincaré . 50

5 Teichmüller's Work on Moduli Space $\mathcal{M}_{g} \ldots 52$ 5.1 Teichmüller's Rigorous Formulation of and Results on Riemann's Moduli Space 52

5.2 Why Did Teichmüller Study Teichmüller Space and Riemann's Moduli Spaces? . . 57

6 Siegel Upper Half Space and Weil's Work on Weil-Petersson Metric . . . . . . . . . . . . 61

7 Grothendieck's Papers at Cartan Seminar . . . 62

8 Baily Compactification, Algebraic Structure on $\mathcal{M}_{g}$, and Torelli Morphism . . . . . . . . 64

9 Mumford's Work on $\mathcal{M}_{g}$ and Moduli Spaces . 67 10 Ahlfors and Bers' Works on Complex Analytic Geometry on Moduli Spaces . . . . . . . . 69 11 Meanings of Moduli in Moduli Spaces . . . . 70 Acknowledgments . . . . . . . . . . . . . 71 References . . . . . . . . . . . . . . . . . . . 71

\section{Introduction}

Mathematics is interesting and complicated. It also has interesting and complicated stories, which are often intriguing. Heroes can be mathematicians, or mathematical concepts and results. But interaction between them is probably more interesting.

In this article, we will tell the story of Riemann's moduli space. There are several reasons for choosing this topic. First, it had an honorable origin in the masterpiece "The theory of Abelian functions" by Riemann in 1857 [65, pp. 79-134]. Second, Riemann's moduli space of Riemann surfaces has been attracting more and more of people's attention, and research on it will probably continue for a long time 


\section{The Story of Riemann's Moduli Space}

\section{by Lizhen $\mathrm{Ji}^{*}$}

\begin{abstract}
Compact Riemann surfaces and projective algebraic curves over $\mathbb{C}$ are two realizations of the same class of objects. Their moduli space was first introduced by Riemann and is one of the central objects of the contemporary mathematics and mathematical physics. In this note, we explain the birth and evolution of Riemann's moduli space by emphasizing several points which might not be so well-known to the general mathematicians and possibly even to some mathematicians who are interested in either complex analytic or algebraic geometric theories of moduli spaces. For example, who was the first to formulate the moduli problem precisely? What is the meaning of moduli spaces? Who was the first to give complex analytic and algebraic variety structures to Riemann's moduli space? Why did they study these problems? How was Riemann's moduli space first used? And why did Riemann choose and use the word moduli? Why did Teichmüller study Teichmüller space? What were Teichmüller's contributions to Riemann's moduli space? What were Teichmüller's contributions to algebraic geometry?
\end{abstract}

\section{Contents}

1 Introduction . . . . . . . . . . . . . . 46

2 Definitions . . . . . . . . . . . . . . 47

3 Riemann's Count of Moduli for Riemann Sur-

faces . . . . . . . . . . . . . . . . 49

* Department of Mathematics, University of Michigan, Ann Arbor, Mich., U.S.A.

E-mail: lji@umich.edu
4 Klein's Booklet on Riemann's Work and the Method of Continuity to Prove the Uniformization Theorem by Klein and Poincaré . 50

5 Teichmüller's Work on Moduli Space $\mathcal{M}_{g} \ldots 52$ 5.1 Teichmüller's Rigorous Formulation of and Results on Riemann's Moduli Space 52

5.2 Why Did Teichmüller Study Teichmüller Space and Riemann's Moduli Spaces? . . 57

6 Siegel Upper Half Space and Weil's Work on Weil-Petersson Metric . . . . . . . . . . . . 61

7 Grothendieck's Papers at Cartan Seminar . . . 62

8 Baily Compactification, Algebraic Structure on $\mathcal{M}_{g}$, and Torelli Morphism . . . . . . . . 64

9 Mumford's Work on $\mathcal{M}_{g}$ and Moduli Spaces . 67 10 Ahlfors and Bers' Works on Complex Analytic Geometry on Moduli Spaces . . . . . . . . 69 11 Meanings of Moduli in Moduli Spaces . . . . 70 Acknowledgments . . . . . . . . . . . . . 71 References . . . . . . . . . . . . . . . . . . . 71

\section{Introduction}

Mathematics is interesting and complicated. It also has interesting and complicated stories, which are often intriguing. Heroes can be mathematicians, or mathematical concepts and results. But interaction between them is probably more interesting.

In this article, we will tell the story of Riemann's moduli space. There are several reasons for choosing this topic. First, it had an honorable origin in the masterpiece "The theory of Abelian functions" by Riemann in 1857 [65, pp. 79-134]. Second, Riemann's moduli space of Riemann surfaces has been attracting more and more of people's attention, and research on it will probably continue for a long time 


\section{The Story of Riemann's Moduli Space}

\section{by Lizhen $\mathrm{Ji}^{*}$}

\begin{abstract}
Compact Riemann surfaces and projective algebraic curves over $\mathbb{C}$ are two realizations of the same class of objects. Their moduli space was first introduced by Riemann and is one of the central objects of the contemporary mathematics and mathematical physics. In this note, we explain the birth and evolution of Riemann's moduli space by emphasizing several points which might not be so well-known to the general mathematicians and possibly even to some mathematicians who are interested in either complex analytic or algebraic geometric theories of moduli spaces. For example, who was the first to formulate the moduli problem precisely? What is the meaning of moduli spaces? Who was the first to give complex analytic and algebraic variety structures to Riemann's moduli space? Why did they study these problems? How was Riemann's moduli space first used? And why did Riemann choose and use the word moduli? Why did Teichmüller study Teichmüller space? What were Teichmüller's contributions to Riemann's moduli space? What were Teichmüller's contributions to algebraic geometry?
\end{abstract}

\section{Contents}

1 Introduction . . . . . . . . . . . . . . 46

2 Definitions . . . . . . . . . . . . . . 47

3 Riemann's Count of Moduli for Riemann Sur-

faces . . . . . . . . . . . . . . . . 49

* Department of Mathematics, University of Michigan, Ann Arbor, Mich., U.S.A.

E-mail: lji@umich.edu
4 Klein's Booklet on Riemann's Work and the Method of Continuity to Prove the Uniformization Theorem by Klein and Poincaré . 50

5 Teichmüller's Work on Moduli Space $\mathcal{M}_{g} \ldots 52$ 5.1 Teichmüller's Rigorous Formulation of and Results on Riemann's Moduli Space 52

5.2 Why Did Teichmüller Study Teichmüller Space and Riemann's Moduli Spaces? . . 57

6 Siegel Upper Half Space and Weil's Work on Weil-Petersson Metric . . . . . . . . . . . . 61

7 Grothendieck's Papers at Cartan Seminar . . . 62

8 Baily Compactification, Algebraic Structure on $\mathcal{M}_{g}$, and Torelli Morphism . . . . . . . . 64

9 Mumford's Work on $\mathcal{M}_{g}$ and Moduli Spaces . 67 10 Ahlfors and Bers' Works on Complex Analytic Geometry on Moduli Spaces . . . . . . . . 69 11 Meanings of Moduli in Moduli Spaces . . . . 70 Acknowledgments . . . . . . . . . . . . . 71 References . . . . . . . . . . . . . . . . . . . 71

\section{Introduction}

Mathematics is interesting and complicated. It also has interesting and complicated stories, which are often intriguing. Heroes can be mathematicians, or mathematical concepts and results. But interaction between them is probably more interesting.

In this article, we will tell the story of Riemann's moduli space. There are several reasons for choosing this topic. First, it had an honorable origin in the masterpiece "The theory of Abelian functions" by Riemann in 1857 [65, pp. 79-134]. Second, Riemann's moduli space of Riemann surfaces has been attracting more and more of people's attention, and research on it will probably continue for a long time 


\section{The Story of Riemann's Moduli Space}

\section{by Lizhen $\mathrm{Ji}^{*}$}

\begin{abstract}
Compact Riemann surfaces and projective algebraic curves over $\mathbb{C}$ are two realizations of the same class of objects. Their moduli space was first introduced by Riemann and is one of the central objects of the contemporary mathematics and mathematical physics. In this note, we explain the birth and evolution of Riemann's moduli space by emphasizing several points which might not be so well-known to the general mathematicians and possibly even to some mathematicians who are interested in either complex analytic or algebraic geometric theories of moduli spaces. For example, who was the first to formulate the moduli problem precisely? What is the meaning of moduli spaces? Who was the first to give complex analytic and algebraic variety structures to Riemann's moduli space? Why did they study these problems? How was Riemann's moduli space first used? And why did Riemann choose and use the word moduli? Why did Teichmüller study Teichmüller space? What were Teichmüller's contributions to Riemann's moduli space? What were Teichmüller's contributions to algebraic geometry?
\end{abstract}

\section{Contents}

1 Introduction . . . . . . . . . . . . . . 46

2 Definitions . . . . . . . . . . . . . . 47

3 Riemann's Count of Moduli for Riemann Sur-

faces . . . . . . . . . . . . . . . . 49

* Department of Mathematics, University of Michigan, Ann Arbor, Mich., U.S.A.

E-mail: lji@umich.edu
4 Klein's Booklet on Riemann's Work and the Method of Continuity to Prove the Uniformization Theorem by Klein and Poincaré . 50

5 Teichmüller's Work on Moduli Space $\mathcal{M}_{g} \ldots 52$ 5.1 Teichmüller's Rigorous Formulation of and Results on Riemann's Moduli Space 52

5.2 Why Did Teichmüller Study Teichmüller Space and Riemann's Moduli Spaces? . . 57

6 Siegel Upper Half Space and Weil's Work on Weil-Petersson Metric . . . . . . . . . . . . 61

7 Grothendieck's Papers at Cartan Seminar . . . 62

8 Baily Compactification, Algebraic Structure on $\mathcal{M}_{g}$, and Torelli Morphism . . . . . . . . 64

9 Mumford's Work on $\mathcal{M}_{g}$ and Moduli Spaces . 67 10 Ahlfors and Bers' Works on Complex Analytic Geometry on Moduli Spaces . . . . . . . . 69 11 Meanings of Moduli in Moduli Spaces . . . . 70 Acknowledgments . . . . . . . . . . . . . 71 References . . . . . . . . . . . . . . . . . . . 71

\section{Introduction}

Mathematics is interesting and complicated. It also has interesting and complicated stories, which are often intriguing. Heroes can be mathematicians, or mathematical concepts and results. But interaction between them is probably more interesting.

In this article, we will tell the story of Riemann's moduli space. There are several reasons for choosing this topic. First, it had an honorable origin in the masterpiece "The theory of Abelian functions" by Riemann in 1857 [65, pp. 79-134]. Second, Riemann's moduli space of Riemann surfaces has been attracting more and more of people's attention, and research on it will probably continue for a long time 


\section{The Story of Riemann's Moduli Space}

\section{by Lizhen $\mathrm{Ji}^{*}$}

\begin{abstract}
Compact Riemann surfaces and projective algebraic curves over $\mathbb{C}$ are two realizations of the same class of objects. Their moduli space was first introduced by Riemann and is one of the central objects of the contemporary mathematics and mathematical physics. In this note, we explain the birth and evolution of Riemann's moduli space by emphasizing several points which might not be so well-known to the general mathematicians and possibly even to some mathematicians who are interested in either complex analytic or algebraic geometric theories of moduli spaces. For example, who was the first to formulate the moduli problem precisely? What is the meaning of moduli spaces? Who was the first to give complex analytic and algebraic variety structures to Riemann's moduli space? Why did they study these problems? How was Riemann's moduli space first used? And why did Riemann choose and use the word moduli? Why did Teichmüller study Teichmüller space? What were Teichmüller's contributions to Riemann's moduli space? What were Teichmüller's contributions to algebraic geometry?
\end{abstract}

\section{Contents}

1 Introduction . . . . . . . . . . . . . . 46

2 Definitions . . . . . . . . . . . . . . 47

3 Riemann's Count of Moduli for Riemann Sur-

faces . . . . . . . . . . . . . . . . 49

* Department of Mathematics, University of Michigan, Ann Arbor, Mich., U.S.A.

E-mail: lji@umich.edu
4 Klein's Booklet on Riemann's Work and the Method of Continuity to Prove the Uniformization Theorem by Klein and Poincaré . 50

5 Teichmüller's Work on Moduli Space $\mathcal{M}_{g} \ldots 52$ 5.1 Teichmüller's Rigorous Formulation of and Results on Riemann's Moduli Space 52

5.2 Why Did Teichmüller Study Teichmüller Space and Riemann's Moduli Spaces? . . 57

6 Siegel Upper Half Space and Weil's Work on Weil-Petersson Metric . . . . . . . . . . . . 61

7 Grothendieck's Papers at Cartan Seminar . . . 62

8 Baily Compactification, Algebraic Structure on $\mathcal{M}_{g}$, and Torelli Morphism . . . . . . . . 64

9 Mumford's Work on $\mathcal{M}_{g}$ and Moduli Spaces . 67 10 Ahlfors and Bers' Works on Complex Analytic Geometry on Moduli Spaces . . . . . . . . 69 11 Meanings of Moduli in Moduli Spaces . . . . 70 Acknowledgments . . . . . . . . . . . . . 71 References . . . . . . . . . . . . . . . . . . . 71

\section{Introduction}

Mathematics is interesting and complicated. It also has interesting and complicated stories, which are often intriguing. Heroes can be mathematicians, or mathematical concepts and results. But interaction between them is probably more interesting.

In this article, we will tell the story of Riemann's moduli space. There are several reasons for choosing this topic. First, it had an honorable origin in the masterpiece "The theory of Abelian functions" by Riemann in 1857 [65, pp. 79-134]. Second, Riemann's moduli space of Riemann surfaces has been attracting more and more of people's attention, and research on it will probably continue for a long time 


\section{The Story of Riemann's Moduli Space}

\section{by Lizhen $\mathrm{Ji}^{*}$}

\begin{abstract}
Compact Riemann surfaces and projective algebraic curves over $\mathbb{C}$ are two realizations of the same class of objects. Their moduli space was first introduced by Riemann and is one of the central objects of the contemporary mathematics and mathematical physics. In this note, we explain the birth and evolution of Riemann's moduli space by emphasizing several points which might not be so well-known to the general mathematicians and possibly even to some mathematicians who are interested in either complex analytic or algebraic geometric theories of moduli spaces. For example, who was the first to formulate the moduli problem precisely? What is the meaning of moduli spaces? Who was the first to give complex analytic and algebraic variety structures to Riemann's moduli space? Why did they study these problems? How was Riemann's moduli space first used? And why did Riemann choose and use the word moduli? Why did Teichmüller study Teichmüller space? What were Teichmüller's contributions to Riemann's moduli space? What were Teichmüller's contributions to algebraic geometry?
\end{abstract}

\section{Contents}

1 Introduction . . . . . . . . . . . . . . 46

2 Definitions . . . . . . . . . . . . . . 47

3 Riemann's Count of Moduli for Riemann Sur-

faces . . . . . . . . . . . . . . . . 49

* Department of Mathematics, University of Michigan, Ann Arbor, Mich., U.S.A.

E-mail: lji@umich.edu
4 Klein's Booklet on Riemann's Work and the Method of Continuity to Prove the Uniformization Theorem by Klein and Poincaré . 50

5 Teichmüller's Work on Moduli Space $\mathcal{M}_{g} \ldots 52$ 5.1 Teichmüller's Rigorous Formulation of and Results on Riemann's Moduli Space 52

5.2 Why Did Teichmüller Study Teichmüller Space and Riemann's Moduli Spaces? . . 57

6 Siegel Upper Half Space and Weil's Work on Weil-Petersson Metric . . . . . . . . . . . . 61

7 Grothendieck's Papers at Cartan Seminar . . . 62

8 Baily Compactification, Algebraic Structure on $\mathcal{M}_{g}$, and Torelli Morphism . . . . . . . . 64

9 Mumford's Work on $\mathcal{M}_{g}$ and Moduli Spaces . 67 10 Ahlfors and Bers' Works on Complex Analytic Geometry on Moduli Spaces . . . . . . . . 69 11 Meanings of Moduli in Moduli Spaces . . . . 70 Acknowledgments . . . . . . . . . . . . . 71 References . . . . . . . . . . . . . . . . . . . 71

\section{Introduction}

Mathematics is interesting and complicated. It also has interesting and complicated stories, which are often intriguing. Heroes can be mathematicians, or mathematical concepts and results. But interaction between them is probably more interesting.

In this article, we will tell the story of Riemann's moduli space. There are several reasons for choosing this topic. First, it had an honorable origin in the masterpiece "The theory of Abelian functions" by Riemann in 1857 [65, pp. 79-134]. Second, Riemann's moduli space of Riemann surfaces has been attracting more and more of people's attention, and research on it will probably continue for a long time 


\section{The Story of Riemann's Moduli Space}

\section{by Lizhen $\mathrm{Ji}^{*}$}

\begin{abstract}
Compact Riemann surfaces and projective algebraic curves over $\mathbb{C}$ are two realizations of the same class of objects. Their moduli space was first introduced by Riemann and is one of the central objects of the contemporary mathematics and mathematical physics. In this note, we explain the birth and evolution of Riemann's moduli space by emphasizing several points which might not be so well-known to the general mathematicians and possibly even to some mathematicians who are interested in either complex analytic or algebraic geometric theories of moduli spaces. For example, who was the first to formulate the moduli problem precisely? What is the meaning of moduli spaces? Who was the first to give complex analytic and algebraic variety structures to Riemann's moduli space? Why did they study these problems? How was Riemann's moduli space first used? And why did Riemann choose and use the word moduli? Why did Teichmüller study Teichmüller space? What were Teichmüller's contributions to Riemann's moduli space? What were Teichmüller's contributions to algebraic geometry?
\end{abstract}

\section{Contents}

1 Introduction . . . . . . . . . . . . . . 46

2 Definitions . . . . . . . . . . . . . . 47

3 Riemann's Count of Moduli for Riemann Sur-

faces . . . . . . . . . . . . . . . . 49

* Department of Mathematics, University of Michigan, Ann Arbor, Mich., U.S.A.

E-mail: lji@umich.edu
4 Klein's Booklet on Riemann's Work and the Method of Continuity to Prove the Uniformization Theorem by Klein and Poincaré . 50

5 Teichmüller's Work on Moduli Space $\mathcal{M}_{g} \ldots 52$ 5.1 Teichmüller's Rigorous Formulation of and Results on Riemann's Moduli Space 52

5.2 Why Did Teichmüller Study Teichmüller Space and Riemann's Moduli Spaces? . . 57

6 Siegel Upper Half Space and Weil's Work on Weil-Petersson Metric . . . . . . . . . . . . 61

7 Grothendieck's Papers at Cartan Seminar . . . 62

8 Baily Compactification, Algebraic Structure on $\mathcal{M}_{g}$, and Torelli Morphism . . . . . . . . 64

9 Mumford's Work on $\mathcal{M}_{g}$ and Moduli Spaces . 67 10 Ahlfors and Bers' Works on Complex Analytic Geometry on Moduli Spaces . . . . . . . . 69 11 Meanings of Moduli in Moduli Spaces . . . . 70 Acknowledgments . . . . . . . . . . . . . 71 References . . . . . . . . . . . . . . . . . . . 71

\section{Introduction}

Mathematics is interesting and complicated. It also has interesting and complicated stories, which are often intriguing. Heroes can be mathematicians, or mathematical concepts and results. But interaction between them is probably more interesting.

In this article, we will tell the story of Riemann's moduli space. There are several reasons for choosing this topic. First, it had an honorable origin in the masterpiece "The theory of Abelian functions" by Riemann in 1857 [65, pp. 79-134]. Second, Riemann's moduli space of Riemann surfaces has been attracting more and more of people's attention, and research on it will probably continue for a long time 


\section{The Story of Riemann's Moduli Space}

\section{by Lizhen $\mathrm{Ji}^{*}$}

\begin{abstract}
Compact Riemann surfaces and projective algebraic curves over $\mathbb{C}$ are two realizations of the same class of objects. Their moduli space was first introduced by Riemann and is one of the central objects of the contemporary mathematics and mathematical physics. In this note, we explain the birth and evolution of Riemann's moduli space by emphasizing several points which might not be so well-known to the general mathematicians and possibly even to some mathematicians who are interested in either complex analytic or algebraic geometric theories of moduli spaces. For example, who was the first to formulate the moduli problem precisely? What is the meaning of moduli spaces? Who was the first to give complex analytic and algebraic variety structures to Riemann's moduli space? Why did they study these problems? How was Riemann's moduli space first used? And why did Riemann choose and use the word moduli? Why did Teichmüller study Teichmüller space? What were Teichmüller's contributions to Riemann's moduli space? What were Teichmüller's contributions to algebraic geometry?
\end{abstract}

\section{Contents}

1 Introduction . . . . . . . . . . . . . . 46

2 Definitions . . . . . . . . . . . . . . 47

3 Riemann's Count of Moduli for Riemann Sur-

faces . . . . . . . . . . . . . . . . 49

* Department of Mathematics, University of Michigan, Ann Arbor, Mich., U.S.A.

E-mail: lji@umich.edu
4 Klein's Booklet on Riemann's Work and the Method of Continuity to Prove the Uniformization Theorem by Klein and Poincaré . 50

5 Teichmüller's Work on Moduli Space $\mathcal{M}_{g} \ldots 52$ 5.1 Teichmüller's Rigorous Formulation of and Results on Riemann's Moduli Space 52

5.2 Why Did Teichmüller Study Teichmüller Space and Riemann's Moduli Spaces? . . 57

6 Siegel Upper Half Space and Weil's Work on Weil-Petersson Metric . . . . . . . . . . . . 61

7 Grothendieck's Papers at Cartan Seminar . . . 62

8 Baily Compactification, Algebraic Structure on $\mathcal{M}_{g}$, and Torelli Morphism . . . . . . . . 64

9 Mumford's Work on $\mathcal{M}_{g}$ and Moduli Spaces . 67 10 Ahlfors and Bers' Works on Complex Analytic Geometry on Moduli Spaces . . . . . . . . 69 11 Meanings of Moduli in Moduli Spaces . . . . 70 Acknowledgments . . . . . . . . . . . . . 71 References . . . . . . . . . . . . . . . . . . . 71

\section{Introduction}

Mathematics is interesting and complicated. It also has interesting and complicated stories, which are often intriguing. Heroes can be mathematicians, or mathematical concepts and results. But interaction between them is probably more interesting.

In this article, we will tell the story of Riemann's moduli space. There are several reasons for choosing this topic. First, it had an honorable origin in the masterpiece "The theory of Abelian functions" by Riemann in 1857 [65, pp. 79-134]. Second, Riemann's moduli space of Riemann surfaces has been attracting more and more of people's attention, and research on it will probably continue for a long time 


\section{The Story of Riemann's Moduli Space}

\section{by Lizhen $\mathrm{Ji}^{*}$}

\begin{abstract}
Compact Riemann surfaces and projective algebraic curves over $\mathbb{C}$ are two realizations of the same class of objects. Their moduli space was first introduced by Riemann and is one of the central objects of the contemporary mathematics and mathematical physics. In this note, we explain the birth and evolution of Riemann's moduli space by emphasizing several points which might not be so well-known to the general mathematicians and possibly even to some mathematicians who are interested in either complex analytic or algebraic geometric theories of moduli spaces. For example, who was the first to formulate the moduli problem precisely? What is the meaning of moduli spaces? Who was the first to give complex analytic and algebraic variety structures to Riemann's moduli space? Why did they study these problems? How was Riemann's moduli space first used? And why did Riemann choose and use the word moduli? Why did Teichmüller study Teichmüller space? What were Teichmüller's contributions to Riemann's moduli space? What were Teichmüller's contributions to algebraic geometry?
\end{abstract}

\section{Contents}

1 Introduction . . . . . . . . . . . . . . 46

2 Definitions . . . . . . . . . . . . . . 47

3 Riemann's Count of Moduli for Riemann Sur-

faces . . . . . . . . . . . . . . . . 49

* Department of Mathematics, University of Michigan, Ann Arbor, Mich., U.S.A.

E-mail: lji@umich.edu
4 Klein's Booklet on Riemann's Work and the Method of Continuity to Prove the Uniformization Theorem by Klein and Poincaré . 50

5 Teichmüller's Work on Moduli Space $\mathcal{M}_{g} \ldots 52$ 5.1 Teichmüller's Rigorous Formulation of and Results on Riemann's Moduli Space 52

5.2 Why Did Teichmüller Study Teichmüller Space and Riemann's Moduli Spaces? . . 57

6 Siegel Upper Half Space and Weil's Work on Weil-Petersson Metric . . . . . . . . . . . . 61

7 Grothendieck's Papers at Cartan Seminar . . . 62

8 Baily Compactification, Algebraic Structure on $\mathcal{M}_{g}$, and Torelli Morphism . . . . . . . . 64

9 Mumford's Work on $\mathcal{M}_{g}$ and Moduli Spaces . 67 10 Ahlfors and Bers' Works on Complex Analytic Geometry on Moduli Spaces . . . . . . . . 69 11 Meanings of Moduli in Moduli Spaces . . . . 70 Acknowledgments . . . . . . . . . . . . . 71 References . . . . . . . . . . . . . . . . . . . 71

\section{Introduction}

Mathematics is interesting and complicated. It also has interesting and complicated stories, which are often intriguing. Heroes can be mathematicians, or mathematical concepts and results. But interaction between them is probably more interesting.

In this article, we will tell the story of Riemann's moduli space. There are several reasons for choosing this topic. First, it had an honorable origin in the masterpiece "The theory of Abelian functions" by Riemann in 1857 [65, pp. 79-134]. Second, Riemann's moduli space of Riemann surfaces has been attracting more and more of people's attention, and research on it will probably continue for a long time 


\section{The Story of Riemann's Moduli Space}

\section{by Lizhen $\mathrm{Ji}^{*}$}

\begin{abstract}
Compact Riemann surfaces and projective algebraic curves over $\mathbb{C}$ are two realizations of the same class of objects. Their moduli space was first introduced by Riemann and is one of the central objects of the contemporary mathematics and mathematical physics. In this note, we explain the birth and evolution of Riemann's moduli space by emphasizing several points which might not be so well-known to the general mathematicians and possibly even to some mathematicians who are interested in either complex analytic or algebraic geometric theories of moduli spaces. For example, who was the first to formulate the moduli problem precisely? What is the meaning of moduli spaces? Who was the first to give complex analytic and algebraic variety structures to Riemann's moduli space? Why did they study these problems? How was Riemann's moduli space first used? And why did Riemann choose and use the word moduli? Why did Teichmüller study Teichmüller space? What were Teichmüller's contributions to Riemann's moduli space? What were Teichmüller's contributions to algebraic geometry?
\end{abstract}

\section{Contents}

1 Introduction . . . . . . . . . . . . . . 46

2 Definitions . . . . . . . . . . . . . . 47

3 Riemann's Count of Moduli for Riemann Sur-

faces . . . . . . . . . . . . . . . . 49

* Department of Mathematics, University of Michigan, Ann Arbor, Mich., U.S.A.

E-mail: lji@umich.edu
4 Klein's Booklet on Riemann's Work and the Method of Continuity to Prove the Uniformization Theorem by Klein and Poincaré . 50

5 Teichmüller's Work on Moduli Space $\mathcal{M}_{g} \ldots 52$ 5.1 Teichmüller's Rigorous Formulation of and Results on Riemann's Moduli Space 52

5.2 Why Did Teichmüller Study Teichmüller Space and Riemann's Moduli Spaces? . . 57

6 Siegel Upper Half Space and Weil's Work on Weil-Petersson Metric . . . . . . . . . . . . 61

7 Grothendieck's Papers at Cartan Seminar . . . 62

8 Baily Compactification, Algebraic Structure on $\mathcal{M}_{g}$, and Torelli Morphism . . . . . . . . 64

9 Mumford's Work on $\mathcal{M}_{g}$ and Moduli Spaces . 67 10 Ahlfors and Bers' Works on Complex Analytic Geometry on Moduli Spaces . . . . . . . . 69 11 Meanings of Moduli in Moduli Spaces . . . . 70 Acknowledgments . . . . . . . . . . . . . 71 References . . . . . . . . . . . . . . . . . . . 71

\section{Introduction}

Mathematics is interesting and complicated. It also has interesting and complicated stories, which are often intriguing. Heroes can be mathematicians, or mathematical concepts and results. But interaction between them is probably more interesting.

In this article, we will tell the story of Riemann's moduli space. There are several reasons for choosing this topic. First, it had an honorable origin in the masterpiece "The theory of Abelian functions" by Riemann in 1857 [65, pp. 79-134]. Second, Riemann's moduli space of Riemann surfaces has been attracting more and more of people's attention, and research on it will probably continue for a long time 


\section{The Story of Riemann's Moduli Space}

\section{by Lizhen $\mathrm{Ji}^{*}$}

\begin{abstract}
Compact Riemann surfaces and projective algebraic curves over $\mathbb{C}$ are two realizations of the same class of objects. Their moduli space was first introduced by Riemann and is one of the central objects of the contemporary mathematics and mathematical physics. In this note, we explain the birth and evolution of Riemann's moduli space by emphasizing several points which might not be so well-known to the general mathematicians and possibly even to some mathematicians who are interested in either complex analytic or algebraic geometric theories of moduli spaces. For example, who was the first to formulate the moduli problem precisely? What is the meaning of moduli spaces? Who was the first to give complex analytic and algebraic variety structures to Riemann's moduli space? Why did they study these problems? How was Riemann's moduli space first used? And why did Riemann choose and use the word moduli? Why did Teichmüller study Teichmüller space? What were Teichmüller's contributions to Riemann's moduli space? What were Teichmüller's contributions to algebraic geometry?
\end{abstract}

\section{Contents}

1 Introduction . . . . . . . . . . . . . . 46

2 Definitions . . . . . . . . . . . . . . 47

3 Riemann's Count of Moduli for Riemann Sur-

faces . . . . . . . . . . . . . . . . 49

* Department of Mathematics, University of Michigan, Ann Arbor, Mich., U.S.A.

E-mail: lji@umich.edu
4 Klein's Booklet on Riemann's Work and the Method of Continuity to Prove the Uniformization Theorem by Klein and Poincaré . 50

5 Teichmüller's Work on Moduli Space $\mathcal{M}_{g} \ldots 52$ 5.1 Teichmüller's Rigorous Formulation of and Results on Riemann's Moduli Space 52

5.2 Why Did Teichmüller Study Teichmüller Space and Riemann's Moduli Spaces? . . 57

6 Siegel Upper Half Space and Weil's Work on Weil-Petersson Metric . . . . . . . . . . . . 61

7 Grothendieck's Papers at Cartan Seminar . . . 62

8 Baily Compactification, Algebraic Structure on $\mathcal{M}_{g}$, and Torelli Morphism . . . . . . . . 64

9 Mumford's Work on $\mathcal{M}_{g}$ and Moduli Spaces . 67 10 Ahlfors and Bers' Works on Complex Analytic Geometry on Moduli Spaces . . . . . . . . 69 11 Meanings of Moduli in Moduli Spaces . . . . 70 Acknowledgments . . . . . . . . . . . . . 71 References . . . . . . . . . . . . . . . . . . . 71

\section{Introduction}

Mathematics is interesting and complicated. It also has interesting and complicated stories, which are often intriguing. Heroes can be mathematicians, or mathematical concepts and results. But interaction between them is probably more interesting.

In this article, we will tell the story of Riemann's moduli space. There are several reasons for choosing this topic. First, it had an honorable origin in the masterpiece "The theory of Abelian functions" by Riemann in 1857 [65, pp. 79-134]. Second, Riemann's moduli space of Riemann surfaces has been attracting more and more of people's attention, and research on it will probably continue for a long time 